Full length article

\title{
Fabrication and characterization of Eri silk fibers-based sponges for biomedical application
}

\author{
Simone S. Silva a,b,*, Nuno M. Oliveira ${ }^{\mathrm{a}, \mathrm{b}}$, Mariana B. Oliveira ${ }^{\mathrm{a}, \mathrm{b}}$, Diana P. Soares da Costa ${ }^{\mathrm{a}, \mathrm{b}}$, \\ Deboki Naskar $^{\text {c }}$, João F. Mano ${ }^{\text {a,b }}$, Subhas C. Kundu ${ }^{\text {c,* }}$, Rui L. Reis ${ }^{\text {a,b }}$ \\ a 3B's Research Group-Biomaterials, Biodegradables and Biomimetics, University of Minho, Headquarters of the European Institute of Excellence on Tissue Engineering \\ and Regenerative Medicine, Avepark - Parque de Ciência e Tecnologia, Zona Industrial da Gandra, 4806-017, Barco GMR, Portugal \\ b ICVS/3B's - PT Government Associate Laboratory, Braga/Guimarães, Portugal \\ ${ }^{\mathrm{c}}$ Department of Biotechnology, Indian Institute of Technology Kharagpur, West Bengal 721302, India
}

\section{A R T I C L E I N F O}

\section{Article history:}

Received 3 June 2015

Received in revised form 29 December 2015

Accepted 4 January 2016

Available online $\mathrm{xxxx}$

\section{Keywords:}

Eri

Samia ricini

Ionic liquids

Genipin

Cartilage regeneration

Fibroin

\begin{abstract}
A B S T R A C T
Cocoon-derived semi-domesticated Eri silk fibers still lack exploitation for tissue engineering applications due to their poor solubility using conventional methods. The present work explores the ability to process cocoon fibers of non-mulberry Eri silk (Samia/Philosamia ricini) into sponges through a green approach using ionic liquid (IL) - 1-buthyl-imidazolium acetate as a solvent. The formation of $\beta$-sheet structures during Eri silk/IL gelation was acquired by exposing the Eri silk/IL gels to a saturated atmosphere composed of two different solvents: (i) isopropanol/ethanol (physical stabilization) and (ii) genipin, a natural crosslinker, dissolved in ethanol (chemical crosslinking). The sponges were then obtained by freeze-drying. This approach promotes the formation of both stable and ordered non-crosslinked Eri silk fibroin matrices. Moreover, genipin-crosslinked silk fibroin sponges presenting high height recovery capacity after compression, high swelling degree and suitable mechanical properties for tissue engineering applications were produced. The incorporation of a model drug - ibuprofen - and the corresponding release study from the loaded sponges demonstrated the potential of using these matrices as effective drug delivery systems. The assessment of the biological performance of ATDC5 chondrocyte-like cells in contact with the developed sponges showed the promotion of cell adhesion and proliferation, as well as extracellular matrix production within 2 weeks of culture. Sponges' intrinsic properties and biological findings open up their potential use for biomedical applications.
\end{abstract}

\section{Statement of Significance}

This work addresses the preparation and characterization of non-mulberry cocoon-derived Eri silk sponges. The insolubility of cocoons-derived non-mulberry silkworms impairs their processability and applications in the healthcare field. We used a green approach with ionic liquids to overcome the lack solubility of such silk fibers. The formation of beta-sheet structures into Eri-based sponges was physically and chemically induced. The sponges were obtained by freeze-drying. The developed structures exhibited flexibility to adapt and recover their shapes upon application and subsequent removal of load, high swelling degree, ability to load an anti-inflammatory drug and to promote its sustained release. They promoted in vitro cellular adhesion, proliferation and extracellular matrix production of a chondrocyte-like cell line, opening up their potential application for biomedical applications.

(c) 2016 Acta Materialia Inc. Published by Elsevier Ltd. All rights reserved.

\footnotetext{
* Corresponding authors at: 3B's Research Group-Biomaterials, Biodegradables and Biomimetics, University of Minho, Headquarters of the European Institute of Excellence on Tissue Engineering and Regenerative Medicine, Avepark - Parque de Ciência e Tecnologia, Zona Industrial da Gandra, 4806-017, Barco GMR, Portugal (S.S. Silva). Department of Biotechnology, Indian Institute of Technology Kharagpur, India (S.C. Kundu).

E-mail addresses: simonesilva@dep.uminho.pt (S.S. Silva), Kundu@hijli.iitkgp. ernet.in (S.C. Kundu).
}

\section{Introduction}

An increasing number of chronic diseases such as diabetes, cancer and rheumatoid arthritis have shown increasing world-wide incidence, affecting people of all ages and genders. In particular, some rheumatic diseases such as osteoarthritis can lead to cartilage degeneration. Such high incidence of chronic and 
aging-related diseases has increased the demand for highperforming biomaterials and therapeutic strategies envisaging cartilage regeneration $[1,2]$. Natural polymers have shown several advantages for biomedical applications due to their biodegradability, biocompatibility and cell-recognition ability [3,4]. Silk fibroinbased materials obtained from mulberry silkworm Bombyx mori have been processed in different forms, including 3D scaffolds, nanoparticles, nanofibers and hydrogels [5-8]. These structures are currently proposed for an extended range of tissue engineering approaches [5-8]. In previous studies silks from non-mulberry silkworms namely Antheraea mylitta (Tropical Tasar), Antheraea assama (Muga), and Samia/Philosamia ricini (Eri) (belonging to Saturniidae family) [9] have been also indicated as good source of raw materials to produce silk-based biomaterials for biomedical applications. The potential applications on the biomedical field of these silk materials is growing, and they are gaining popularity as a new promising group of biomaterials for tissue engineering applications [9-14]. Current research on non-mulberry Eri shows the potential of using such fibroin protein for the preparation of different 2D and 3D biomaterial matrices, both for in vitro and in vivo applications in biomedical engineering fields [14]. Eri silk production is cost effective as no agronomic practices are required for their host plant cultivation [14]. Also, Eri silk-based material could be a good alternative natural biomaterial, as compared to silk-based materials prepared using other silk varieties.

Structurally, Eri silk has a primary structure composed of about 100 repeats of alternating polyalanine and glycine domains [15]. Moreover, the proportion of glycine residues is higher in B. mori, which is mainly composed of glycine, alanine and serine residues $[15,16]$, while the content of alanine residues is greater in S. ricini [14]. The differences in the biochemical composition of Eri silk and B. mori may lead to changes in physical and mechanical properties of the native and silk fibroin derivatives, which may be useful in the design of novel biomaterials.

The present work addresses the processability of cocoonderived non-mulberry Eri silk fibers into porous matrices. For that purpose, we have used a green approach employing an ionic liquid (IL)-1-buthyl-3-methyl imidazolium acetate (BMIMAc) - as a solvent, to overcome the lack of solubility of Eri cocoon silk fibers. In most of the reports concerning Eri silk, fibroin is isolated from the silk glands of 5th instar larvae. However, the application of Eri cocoons as a fibroin source is still relatively unexplored due to the lack of solubilization protocols using conventional solvents $[14,17]$. Earlier works emphasized the use of certain ionic liquids (ILs) in the dissolution and processing of protein-based materials from cocoons [18-21]. Findings demonstrate that IL can be ideal to overcome the insolubility of fibroin from the cocoon-derived silks. Both mulberry and non-mulberry silk fibers, namely $B$. mori and A. mylitta, are amenable to be dissolved in ILs, allowing for their processing into films, fibers, scaffolds and sponges $[19,20]$. Previously, it was reported that the biomaterials produced using ILs as solvent or reaction media exhibit attractive physical, mechanical properties, and biological behavior targeting tissue regeneration approaches. Different biomedical applications for such systems were suggested, namely for skin and cartilage regeneration $[19,20]$. Few reports suggest the use of ILs as a solvent for silks namely, Antheraea assamensis silk (Muga) and A. mylitta silk (Tropical Tasar) [19,22]. To the best of our knowledge, there is no report on the use of BMIMAc for the dissolution and processing of Eri silk into sponges. We suggest the use of BMIMAc as a solvent $[19,20]$ to overcome the lack of solubility of cocoon-derived Eri silk fibers. With this strategy we aim at exploring the possible advantages of using silk cocoon-derived Eri fibers as alternative fibroin sources, namely for healthcare applications. With this purpose in mind, we propose the gelation of Eri silk/BMIMAc solution using physical stabilization combined with chemical crosslinking, and the shaping of sponges using the freeze-drying technique. Genipin is a natural crosslinking agent obtained from the fruits of Gardenia jasminoides, showing low cytotoxicity and currently employed to crosslink chitosan, gelatin, silk and other proteins [7,23-25]. Genipin-crosslinked silk matrices deriving from B. mori show improvement of the mechanical properties, and good cellular behavior $[7,25]$. These features suggest that genipin can play a role as an efficient and biocompatible agent for the crosslinking of silk materials deriving from different sources for biomedical applications. Interestingly, previous studies indicate that genipin crosslinking induces the formation of $\beta$-sheet structures on silk fibroin derived from $B$. mori [6,7].

In this work we investigate the ability to tune the physical, mechanical and biological properties of the developed silk fibroin sponges using an IL, BMIMAc, for dissolving non-mulberry Eri silk fibers. ATDC5 chondrocyte-like cells are employed to evaluate adhesion, spreading and extracellular matrix formation of cells related to cartilage tissue onto the developed sponges.

\section{Materials and methods}

\subsection{Materials}

Silk cocoons of the non-mulberry silkworm S. ricini (white variety) were collected from IIT Kharagpur, West Bengal, India. The ionic liquid (IL), 1-butyl-3-methyl imidazolium acetate (BMIMAc), obtained from Sigma Aldrich (St. Louise USA) was chosen as solvent and used without further purification. Genipin was purchased from Wako Chemicals (USA). All other chemicals were reagent grade and were used as received.

\subsection{Methods}

\subsubsection{Eri silk protein fibroin $(E F)$ isolation}

Eri Silk protein fibers were obtained from the silk cocoons of the non-mulberry semi domesticated silkworm S. ricini as previously described [14]. Briefly, cocoons were cut into small pieces and boiled in $1 \mathrm{~g} / 10 \mathrm{ml}$ of $0.02 \mathrm{M} \mathrm{Na}_{2} \mathrm{CO}_{3}$ for one hour. Afterward, the material was washed with deionised water several times to remove the sericin and sodium carbonate from the silk fibers. The degummed fibers were then dried at room temperature.

\subsubsection{Preparation of the Eri silk/IL solutions, cross-linking reactions and formation of sponges}

The Eri silk fibroin sponges were prepared by dissolving the degummed fibers of $S$. ricini cocoons in BMIMAc at $90-95{ }^{\circ} \mathrm{C}$ in a concentration of $10 \%(\mathrm{w} / \mathrm{v})$. The system was kept under stirring for $6 \mathrm{~h}$. After dissolution, the fiber/IL solutions were transferred to specific silicone molds, which were placed over superamphiphobic surfaces prepared as previously described [26,27] with minor changes. Briefly, the glass slides were held above the flame of a paraffin candle until reaching a homogeneous carbon layer on the glass surface. Then, the glass slides were placed in a desiccator with ammonium aqueous solution (30-33\% v/v) and tetraethylorthosilicate (TEOS) (StbÖer reaction) in a ratio of $1: 1$ for $24 \mathrm{~h}$. On this step, by chemical vapor deposition, a silica layer was formed on the surface of the glass slides. Further, the glass slides were submitted to a thermal treatment at $600{ }^{\circ} \mathrm{C}$ for $2 \mathrm{~h}$ to remove carbon from the surface structure. Finally, the samples were placed into a desiccator with $1 \mathrm{H}, 1 \mathrm{H}, 2 \mathrm{H}, 2 \mathrm{H}$-perfluorodecyltrie thoxysilane (97\%) (without immersion) for 3 days to reach the necessary repellence for hydrophilic and hydrophobic solvents [28]. The gelation/structural stabilization of the materials was processed in a saturated atmosphere composed of isopropanol/ethanol vapor (non-solvent) at room temperature (RT) for 2 days. To create cross- 
linked gels, the isopropanol/ethanol vapor was replaced by a genipin solution. Genipin was dissolved in ethanol at a concentration of $10 \mathrm{mM}$, and the solution was incubated in a desiccator at RT for $24 \mathrm{~h}$ under vacuum. The IL removal was made through immersion of the gels in isopropanol/ethanol for 3 days. The non-solvent was changed periodically and the aliquots were collected for conductivity analysis. The conductivities of the aliquots of isopropanol/ethanol were measured using a conductivimeter (INOLAB, Multi level 3 ) with a Sonda WTW TetraCon 325. At the end of the process, both non- and crosslinking gels were washed with distilled water. The molded gels were then frozen at $-20^{\circ} \mathrm{C}$ for at least $2 \mathrm{~h}$ and at $-80^{\circ} \mathrm{C}$ overnight, followed by freeze-drying for 2 days. Throughout the paper, the non- and cross-linked Eri fibroin silk sponges will be referred as EF and EFG, respectively.

\subsubsection{Determination of the crosslinking degree}

The crosslinking degree for the samples was determined using the ninhydrin assay [29,30]. The samples were first lyophilized for $24 \mathrm{~h}$, and then weighed. Subsequently, the lyophilized sample ( $3 \mathrm{mg}$ ) was heated with a ninhydrin solution $(2 \%, \mathrm{w} / \mathrm{v})$ at $100^{\circ} \mathrm{C}$ for $20 \mathrm{~min}$. After this step, the optical absorbance of the solution was recorded with a spectrophotometer (Bio-Rad SmartSpec $\left.^{\mathrm{TM}} 3000, \mathrm{CA}, \mathrm{USA}\right)$ at $570 \mathrm{~nm}$. Glycine solutions at various previously known concentrations were used as a standard. After heating the samples in the ninhydrin solution, the number of free amino groups in the test sample is proportional to the optical absorbance of the solution. The triplicate analysis was performed for each sample. The degree of crosslinking of sample was then calculated following the Eq. (1):

\section{Degree of cross-linking}

$$
=\frac{[(\mathrm{NH} \text { reactive amine }) \text { fresh }-(\mathrm{NH} \text { reactive amine }) \text { fixed }]}{(\mathrm{NH} \text { reactive amine }) \text { fresh } \times 100}
$$

where "fresh" is the mole fraction of free $\mathrm{NH}_{2}$ in non-crosslinked samples and "fixed" is mole fraction of free $\mathrm{NH}_{2}$ remaining in cross-linked samples.

\subsubsection{Drug loading and in vitro drug release studies}

Ibuprofen was used as a model drug to investigate the loading and release ability of the sponges. The impregnation of ibuprofen in the samples was performed through overnight immersion of the samples in a $10 \mathrm{mg} / \mathrm{ml}(\mathrm{w} / \mathrm{v})$ solution of ibuprofen in pure ethanol. Afterward, the impregnated samples were divided into two sets of samples: (i) washing step with ethanol and distilled water, frozen at $-80^{\circ} \mathrm{C}$ for $4 \mathrm{~h}$ and freeze-drying for 3 days or (ii) crosslinking through immersion in a solution of $10 \mathrm{mM}$ genipin in pure ethanol, for $48 \mathrm{~h}$. The samples described in (ii) were further washed with ethanol and distilled water, frozen at $-80^{\circ} \mathrm{C}$ for $4 \mathrm{~h}$ and freeze-dried for 3 days. Such treatments were performed with the goal of controlling the drug release from the samples. Ibuprofen-loaded sponges were weighed and suspended in $5 \mathrm{ml}$ of phosphate buffered solution (PBS). The samples were immersed in a thermostatic water bath at $37^{\circ} \mathrm{C}$ with agitation $(60 \mathrm{rpm})$. Aliquots of $1 \mathrm{ml}$ were withdrawn at predetermined time intervals and the same volume of fresh medium was added to the suspension. The samples were analyzed by UV-Vis spectroscopy at $263 \mathrm{~nm}$ in a microplate reader (Synergy HT, Bio Tek Instruments, USA). The experiments were performed in triplicate, and the results are presented as the average of three measurements and their respective standard deviation. The concentration of ibuprofen was calculated using a standard curve (concentrations ranging from 0.0 to $72 \mu \mathrm{g} /$ $\mathrm{ml}$ ), relating the amount of released ibuprofen with the intensity of light absorbance.

\section{Physicochemical and in vitro biological characterization}

\subsection{Fourier transform infrared spectroscopy (FTIR)}

The infrared spectra were recorded using a Shimadzu-IR Prestige 21 spectrometer in the spectral region of $4000-650 \mathrm{~cm}^{-1}$ with a resolution of $2 \mathrm{~cm}^{-1}$ at 32 scans. The samples were powdered, mixed with $\mathrm{KBr}$, and processed into pellets. Fourier selfdeconvolution (FSD) of the infrared spectra covering the amide I region (1595-1705 $\mathrm{cm}^{-1}$ ) was performed using Origin Software. To measure the relative areas of the amide I components FSD spectra were curve fitted to Gaussian line shape profiles. The deconvoluted amide I spectra area was normalized and the relative areas of the single bands were used to determine the fraction of the secondary structural elements. The band assignments and the detailed procedure to determine $\beta$-sheet crystallinity were described previously by Hu et al. [31].

\subsection{Scanning electron microscopy (SEM)}

The morphology of the samples, after being coated with a conductive gold layer, was observed using a NanoSEM-FEI Nova 200 with an integrated microanalysis X-ray system (EDS - energy dispersive spectrometer).

\subsection{Micro-computed tomography $(\mu-C T)$}

The morphological structure and the calculation of the morphometric parameters that characterize the samples were evaluated by micro-computed tomography (micro-CT). Scanco 20 equipment (Skyscan 1702, Belgium) was used with penetrative X-rays of $58 \mathrm{keV}$ and $130 \mu \mathrm{A}$, in high resolution mode with a pixel size of $11 \mu \mathrm{m}$. The morphological parameters were determined by the analysis of 2D images of the matrices using CT analyzer software (version 1.5, SkyScan). 2D cross-sections of the structure were visualized using DataViewer software (version 1.4.4 64-bit, SkyScan) and the 3D reconstructions were built using CTVox software (version 2.3.0 r810, SkyScan).

\subsection{Dynamic mechanical analysis (DMA)}

Viscoelastic measurements of the EF and EFG sponges were performed using a TRITEC2000B DMA from Triton Technology (UK) in the compressive mode. To evaluate the recovery ability of both EF and EFG sponges (regarding their viscoelastic behavior) after a high deformation, DMA was performed with the samples immersed in PBS at $37^{\circ} \mathrm{C}$. First, both sponges were initially hydrated in PBS for $1 \mathrm{~h}$. Then, they were analyzed for their storage modulus $\left(E^{\prime}\right)$ and damping properties $(\tan \delta)$ before and after being compressed at $360 \mathrm{kPa}$ for $30 \mathrm{~s}$. After the compression step with $360 \mathrm{kPa}$, the sponges were left to recover in phosphate-buffered saline (PBS) for $5 \mathrm{~min}$. In each step, the changes on the geometry of the samples were measured. The sponges were always analyzed under hydrated conditions through immersion of the samples in a Teflon reservoir containing PBS. They were used during all analysis periods. After equilibration at $37^{\circ} \mathrm{C}$, the DMA spectra were obtained from a frequency scan of $0.1-10 \mathrm{~Hz}$. The experiments were performed under constant strain amplitude, corresponding to approximately $1 \%$ of the height of the samples. The average value and standard deviation obtained from four tests are reported for each sample.

Recovery testing upon mechanical compression of the Eri silk fibroin (EF) and crosslinked Eri silk fibroin (EFG) scaffolds was performed in two different stages: (i) immediately after their processing by freeze-drying and (ii) after their culture with ATDC5 cells during 21 days. 


\subsection{Swelling}

Swelling tests were performed by immersing all sponges in PBS for up to 21 days. All experiments were conducted in triplicate. The weight of the swollen samples was measured after removing excess surface water by gently tapping the surface with filter paper. Water uptake was determined by differences between the swollen state (after equilibration and eventual degradation or partial solubilization of the samples) and dried weight. The weight loss of the sponges after 60 days under the above conditions was also calculated based on the difference between the wet and dry weight of the samples.

\subsection{Cell seeding and culture on sponges}

Before testing, the samples were sterilized in an ethylene oxide atmosphere. In vitro cell tests were performed using a cell suspension of ATDC5 chondrocytes-like cells (mouse 129 teratocarcinoma AT805 derived, ECACC, UK). ATDC5 cells were used at passage 25 in a concentration of $3.5 \times 10^{5}$ cells per sponge (with an approximate volume of $0.125 \mathrm{~mm}^{3}$ ). Triplicates were made for each sample. The cells-sponge constructs were incubated at $37^{\circ} \mathrm{C}$ in a humidified 95\% air-5\% $\mathrm{CO}_{2}$ atmosphere and maintained for $1,7,14$ and 21 days. Cells were cultured in Dulbecco's Modified Eagle's Medium-F12 Ham (DMEM-F12, Gibco, UK) supplemented with $10 \%$ fetal bovine serum (FBS Gibco, UK) and 1\% antibiotic/antimycotic (Gibco, UK). Culture medium was replaced twice a week. After 1, 7, 14 and 21 days of culture, the medium was removed. The samples were washed with a PBS solution, fixed with $10 \%$ formalin solution (Sigma, USA) and assessed for MTS test SEM analysis, DNA quantification, and confocal analysis.

The cell morphology and spreading of ATDC5 seeded EF and EFG sponges were monitored by confocal laser scanning microscopy (CLSM). For this purpose, after each culturing period, the cellssponge constructs were fixed in a $10 \%$ formalin solution (Sigma, USA) for $30 \mathrm{~min}$. Then, the samples were incubated with $3 \%$ bovine serum albumin (BSA) for $30 \mathrm{~min}$. The cells were permeabilized using $0.1 \%$ Triton $\mathrm{X}-100$ in PBS for $5 \mathrm{~min}$, incubated with phalloidin-TRITC for $20 \mathrm{~min}$ at room temperature. The samples were then washed with PBS and staining with $5 \mu \mathrm{g} / \mathrm{ml}$ Hoechst33342 was performed for $30 \mathrm{~min}$. Fluorescence images from stained constructs were obtained using a confocal laser scanning microscope (Leica TCS SP8).

The metabolic activity of the ATDC5 chondrocyte-like cells seeded on the EF and EFG sponges was evaluated using the CellTiter $96^{\circledR}$ AQueous One Solution Cell Proliferation Assay (MTS, Promega). Briefly, cells-sponge constructs were placed in a mixture containing serum-free culture medium and MTS reagent at a 5:1 ratio and incubated for $3 \mathrm{~h}$, at the end of which the optical density was read $(\mathrm{OD}=490 \mathrm{~nm})$.

ATDC5 cell proliferation onto EF and EFG sponges was determined using a fluorimetric double strand DNA (dsDNA) quantification kit (PicoGreen, Molecular Probes, Invitrogen, UK). For this purpose, samples collected at 14, 21 and 28 days were transferred into $1.5 \mathrm{ml}$ microtubes containing $1 \mathrm{ml}$ of ultra-pure water. ATDC5 cells-sponge constructs were incubated for one hour at $37^{\circ} \mathrm{C}$ in a water-bath and then stored in a $-80^{\circ} \mathrm{C}$ freezer until testing. Prior to the dsDNA quantification, the constructs were thawed and sonicated for $15 \mathrm{~min}$. The samples and standards (ranging between 0 and $2 \mathrm{mg} \mathrm{ml}^{-1}$ ) were prepared and mixed with a PicoGreen solution in a 200:1 ratio, and placed on an opaque 96 well plate. Triplicates were made for each sample or standard. The plate was incubated for $10 \mathrm{~min}$ in the dark and fluorescence was measured on a microplate ELISA reader (BioTek, USA) using an excitation of $485 / 20 \mathrm{~nm}$ and an emission of $528 / 20 \mathrm{~nm}$. A standard curve was prepared, and sample DNA values were read off from the standard graph.
Glycosaminoglycans (GAGs) quantification assay [32] was used to detect chondrogenic ECM formation after 14, 21 and 28 days of culture. GAGs standards were obtained by preparing a chondroitin sulfate solution ranging from 0 and $30 \mathrm{mg} \mathrm{ml}^{-1}$. In each well of a 96-well plate, samples or standards were added in triplicates and then the dimethylmethylene blue reagent (DMB, Sigma-Aldrich, USA) was added and mixed. The optical density (OD) was measured immediately at $525 \mathrm{~nm}$ on a microplate ELISA reader. A standard curve was created, and GAGs sample values were read off from the standard graph.

\subsection{Statistical analysis}

All quantitative experiments are run in triplicate and results are expressed as a mean \pm standard deviation for $n=3$. Statistical analysis of the data was conducted using two-way ANOVA with Bonferroni's post test by using GraphPadPrism version 5.0 for Windows (GraphPad Software, San Diego, http://www.graphpad.com). Differences between the groups with $p<0.05$ were considered to be statistically significant.

\section{Results and discussion}

The preparation of Eri silk sponges started with the dissolution of degummed Eri (S. ricini) silk fibers in BMIMAc (Fig. 1A). Further on, the gelation of the Eri silk fibroin (EF)/IL solution occurred inside silicone molds using two different approaches: (i) crystallization using ethanol/isopropanol (1:1) vapor, and (ii) combination of crystallization and chemical crosslinking using genipin dissolved in ethanol in order to process non-and crosslinked matrices (EF and EFG respectively). Goujoun et al. observed that the treatment of $A$. assamensis/BMIMAc solution using an ethanol/isopropanol mixture as coagulating solvent resulted in a film with higher $\beta$-sheet content as compared to other solvents used in the study [22]. Hence, we hypothesize that the use the isopropanol/ ethanol could be a good choice to promote the formation of $\beta$ sheets on EF sponges. Our present investigation suggests that the solubility of genipin decreased when isopropanol/ethanol was used. Genipin was consequently dissolved in ethanol. The dissolution/gelation of silk fibroin using ILs occurs through molecular structural rearrangement of the chains [19]. In our approach, the viscous Eri silk/BMIMAc solutions were put under vacuum, resulting in the formation of a gel, which was molded into silicone molds placed over super-amphiphobic surfaces. These surfaces are characterized by high water and some organic solvents repellence due to its surface roughness and low surface energy [26,27]. These surface characteristics avoided the flow out of the Eri silk/BMIMAc solutions from the cylindrical molds during the gelation process. A silk-based biomaterial requires a more ordered crystalline structure to provide their stability. The induction of $\beta$-sheet crystallinity in $B$. mori-derived fibroin is achieved by treatment with aqueous alcoholic solutions [22]. However, the use of water vapor and/or organic saturated atmosphere in the crystallization of fibroin is relatively new [10,33]. Herein, we hypothesize that the solvent composition of the atmosphere associated with exposure time may provide valuable paths to tune the properties of silk for biomedical purposes. It was observed that Eri silk/BMIMAc gelation took at least 2 days under isopropanol/ethanol atmosphere and $24 \mathrm{~h}$ under genipin vapor. In both cases, rigid gels were produced. During the exposure to vapor, a gradual protein gelation and physical/chemical chain crosslinking may occur simultaneously. In the chemical reaction between genipin and the $B$. mori-derived silk fibroin macromolecules, inter- and intramolecular covalent bonds are formed resulting in stable matrices [7]. Subsequently, the BMIMAc removal was carried out through immersion of the gels in ethanol. 


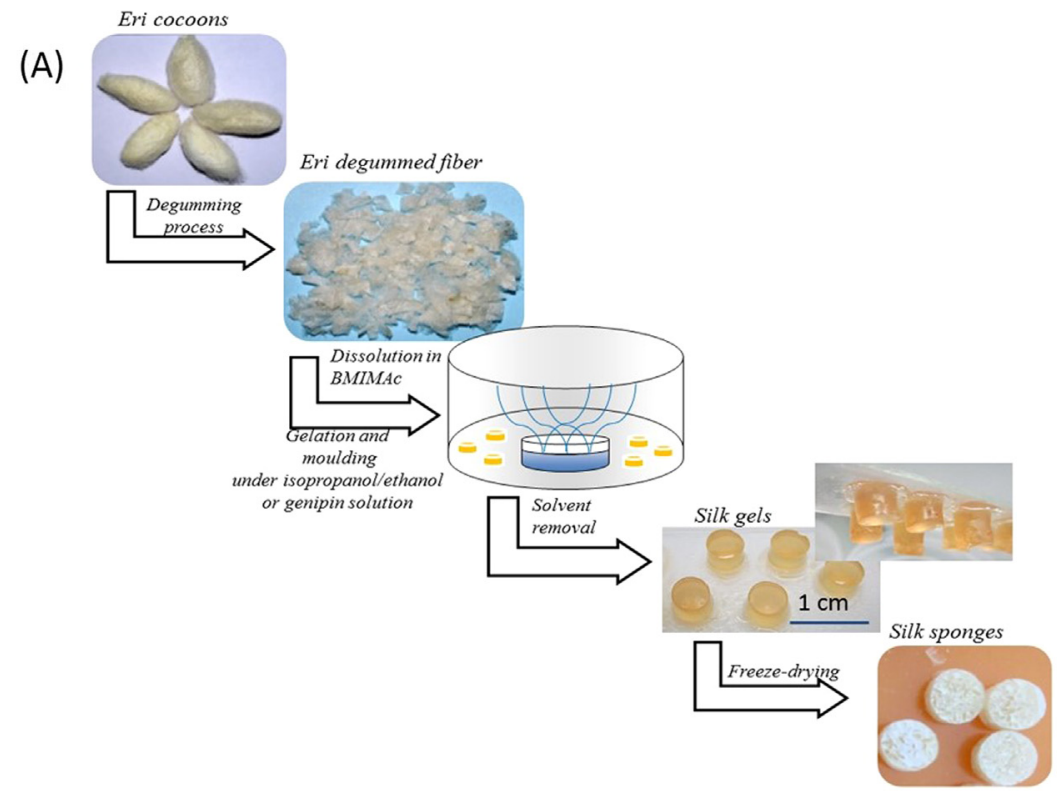

(B)

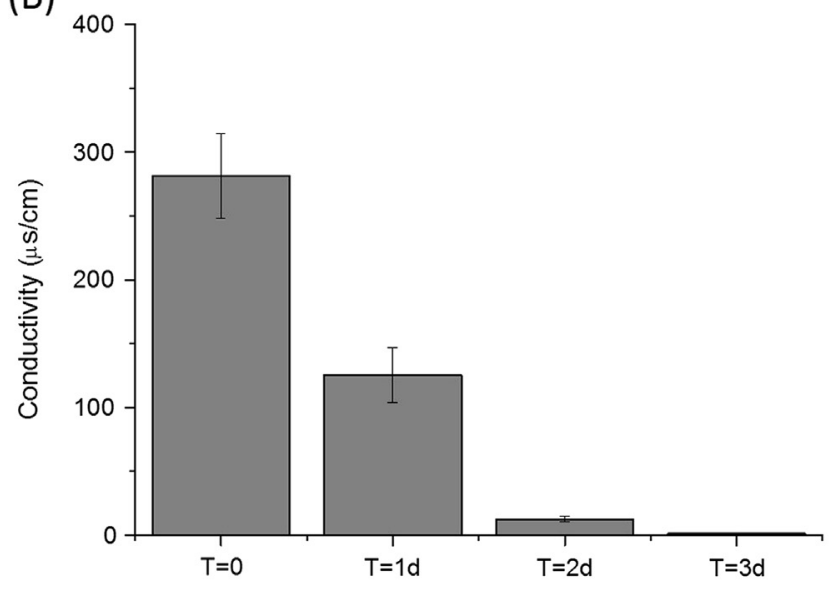

(C)

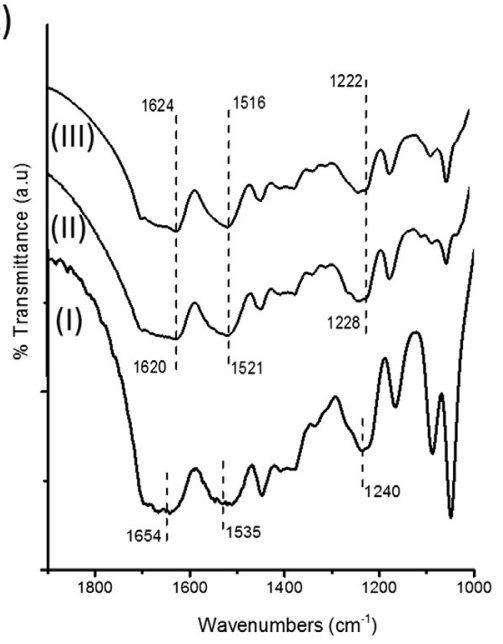

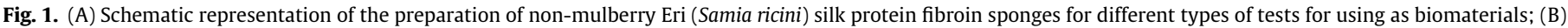

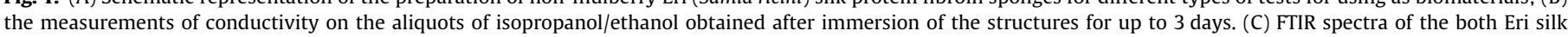
fibroin sponges, (I) Eri degummed fiber, (II) non-crosslinked Eri sponge (EF) and (III) Eri crosslinked sponge (EFG).

Since ILs have an excellent ionic conductivity above their decomposition temperature [34], the elimination of the solvent was monitored through conductivity measurements. The progressive decrease in conductivity during the extraction process indicated the efficiency of the IL removal (Fig. 1B). It is expected that BMIMAc is removed from the Eri hydrogels when the conductivity of the ethanol aliquots dropped below $1.5 \mu \mathrm{S} / \mathrm{cm}$. This also indicated that the fabricated hydrogels have a low level of toxicity. Previous work showed that continuous extraction cycles of IL with ethanol produced bio-matrices with very low cytotoxicity and positive biological responses $[19,20,35,36]$.

Macroscopic (morphology, porosity, mechanical properties) and microscopic (molecular arrangement, crosslinking degree, silk fibroin crystallinity) features of the sponges were considered in this study. These aspects are discussed in the next sections.

The infrared spectra (Fig. 1C) in the range of $1900-1000 \mathrm{~cm}^{-1}$ of the Eri degummed fiber and solvent vapor-treated EF and EFG matrices were used to investigate the structural changes. The degummed fibers exhibited peaks centered at $1654 \mathrm{~cm}^{-1}$,
$1535 \mathrm{~cm}^{-1}$, and $1240 \mathrm{~cm}^{-1}$ attributed to the typical dominant beta sheet along with turn and random coil conformation (Fig. 1C-I) [37-41]. When comparing the degummed fiber (Fig. 1C-I) with spectra EF and EFG in Fig. 1C(II-III), it was observed a shift for $1620-1625 \mathrm{~cm}^{-1}$ (amide I), $1516-1521 \mathrm{~cm}^{-1}$ (amide II) and $1228-1222 \mathrm{~cm}^{-1}$ (amide III), which are associated with intermolecular beta-sheet bands [38]. The formation of crystalline structures was successfully achieved independently of the type of solvent vapor used. In particular, the use of ethanol vapor with dissolved genipin not only induced the formation of $\beta$-sheet structures, but also promoted the formation of an inter-intramolecular network into EF chains. This data is in line with previous studies suggesting that conformational transition occurs from random coil to $\beta$-sheet through structural rearrangement of chains to form covalent bonds [6,7]. By analyzing curve fitting of the FTIR spectra (data not shown) of the amide I region between 1595 and $1705 \mathrm{~cm}^{-1}$ for both sponges, the amount of secondary structure in EF and EFG was estimated. The mentioned areas were chosen since they are related to intermolecular $\beta$-sheet bands [19]. The 
results showed that non-crosslinked fibroin chains (EF samples) have a $38.1 \%$ content of $\beta$-sheet structures, while the formation of such structures increased to $48.7 \%$ after genipin crosslinking (EFG samples).

A ninhydrin assay was performed to evaluate the crosslinking degree of the scaffolds. EFG showed a crosslinking degree of $52.3 \pm 1.7 \%$. Although the genipin reaction on Eri silk is currently unknown, the mechanism may be similar to that observed for amino-group containing compounds [24,42]. Some authors suggested that the genipin preferentially reacts with the amino acids lysine and arginine of certain proteins [7]. Pal et al. [14] reported that the amino acid composition of Eri silk had $4.96 \mathrm{~mol} \%$ of arginine and $0.48 \mathrm{~mol} \%$ of lysine. Both the amino acids (lysine and arginine) are present in the non-crystalline region of silk [43]. The crosslinking process promoted by the genipin treatment occurred on these regions, which indirectly initiates the rearrangement of the whole structure triggering ultimately $\beta$-sheet formation.

\subsection{Morphology features}

The SEM images (Fig. 2A) of the cross-sections indicated that both EF and EFG have an open structure, with pore sizes between 50 and $200 \mu \mathrm{m}$. Also, a quantitative analysis of the 3D morphometric parameters that characterize the silk-based matrices was performed by micro-CT analysis. The 3D images of EF and EFG matrices obtained from digital geometry processing from a series of two-dimensional X-ray images are illustrated in Fig. 2B. Through these images the pore size distribution, porosity and interconnectivity were quantified (Table 1 ). The data revealed that the sponges have an interconnected porous structure, with pore sizes ranging between 60 and $500 \mu \mathrm{m}$, while the mean pore sizes were $224 \mu \mathrm{m}$ and $287 \mu \mathrm{m}$ for EF and EFG, respectively. The pore size distribution of the sponges (Fig. 2C) presented values ranging from 60 to $580 \mu \mathrm{m}$. While EFG sponges showed a wide pore size distribution, EF sponges showed a narrower distribution (Fig. 2C). The
Table 1

Values of mean pore size, interconnectivity and porosity for Eri silk fibroin (EF) and crosslinked Eri silk fibroin (EFG) sponges.

\begin{tabular}{lcll}
\hline Sample & Mean pore size $(\mu \mathrm{m})$ & Interconnectivity $(\%)$ & Porosity $(\%)$ \\
\hline EF & $224.6 \pm 11.7$ & $65.4 \pm 6.7$ & $60.9 \pm 3.1$ \\
EFG & $287.1 \pm 1.9$ & $63.6 \pm 9.1$ & $66.1 \pm 0.3$ \\
\hline
\end{tabular}

different freezing temperatures used for both materials as well as the presence of a crosslinked network of the silk chains (EFG) could affect the porosity, pore size, arrangement and distribution [44]. These factors could explain the observed differences. The observed features of both sponges are adequate for cell migration. Earlier work showed that the optimal pore size for tissue engineering scaffolds should be in the range $150-500 \mu \mathrm{m}$ [45] for the accommodation of the cells for a given tissue. Another parameter needed is high porosity (typically 60-90\%) [46]. The developed sponges fulfill both the requirements (Table 1 ).

\subsection{Mechanical and viscoelastic properties}

To determine the mechanical and viscoelastic nature of the sponges, DMA experiments were performed with the samples immersed in PBS at $37^{\circ} \mathrm{C}$, before and after a compression step. In such compression step, the samples were compressed once using a pressure of $360 \mathrm{kPa}$ during $30 \mathrm{~s}$, and their mechanical and viscoelastic properties were measured immediately after their recovery in PBS. Both storage modulus $\left(E^{\prime}\right)$ and damping properties $(\tan \delta)$ are shown in Fig. 3A. The DMA results show a statistically significant $(p<0.05)$ higher $E^{\prime}$ for EF compared to EFG, right after processing (i.e. previously to the application of the compression pressure): at the frequency of $1 \mathrm{~Hz}$, EF sponges presented a storage modulus of $E^{\prime}$ of $797 \pm 59 \mathrm{kPa}$, while EFG sponges showed an $E^{\prime}$ of $233 \pm 51 \mathrm{kPa}$. After being submitted to crosslinking reactions including reactions with genipin - polymeric materials typically

\section{(A)}
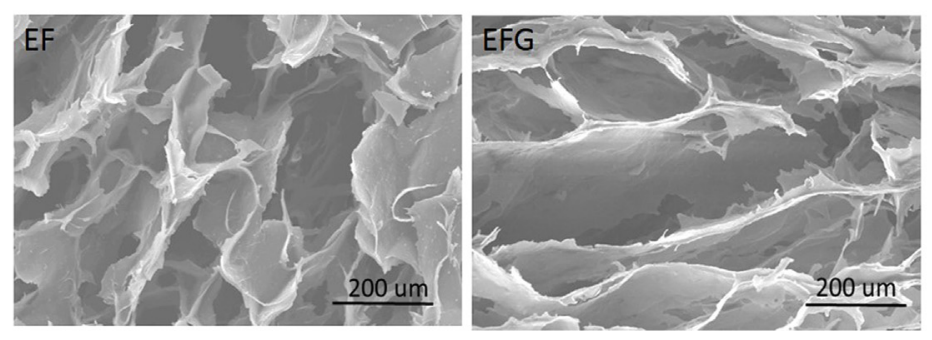

(B)
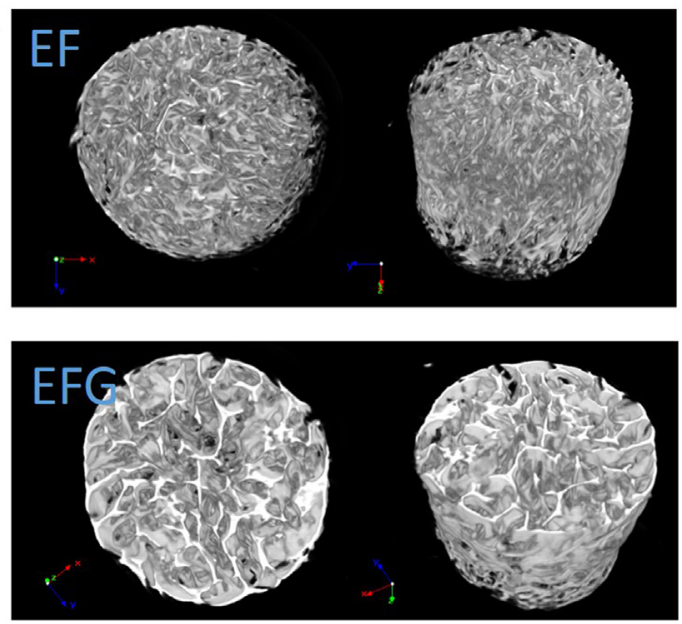

(C)

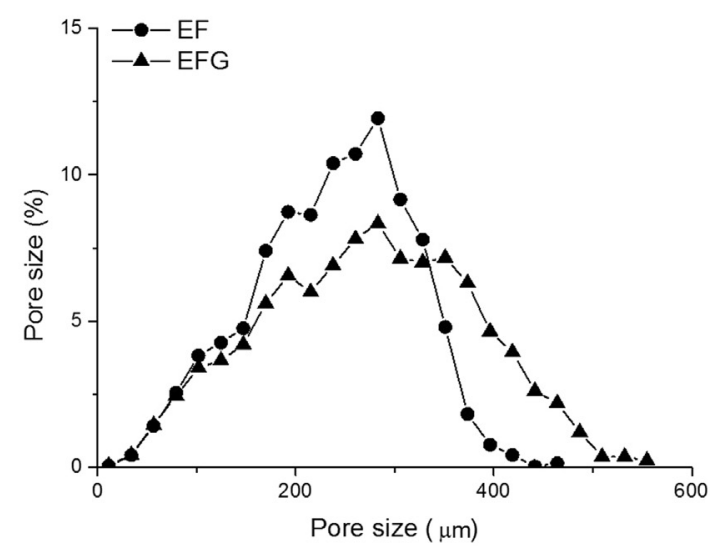

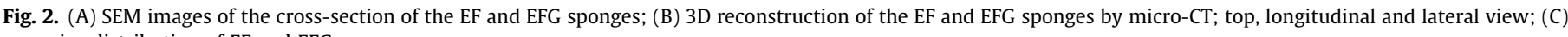
pore size distribution of EF and EFG sponges.

Please cite this article in press as: S.S. Silva et al., Fabrication and characterization of Eri silk fibers-based sponges for biomedical application, Acta Biomater. (2016), http://dx.doi.org/10.1016/j.actbio.2016.01.003 


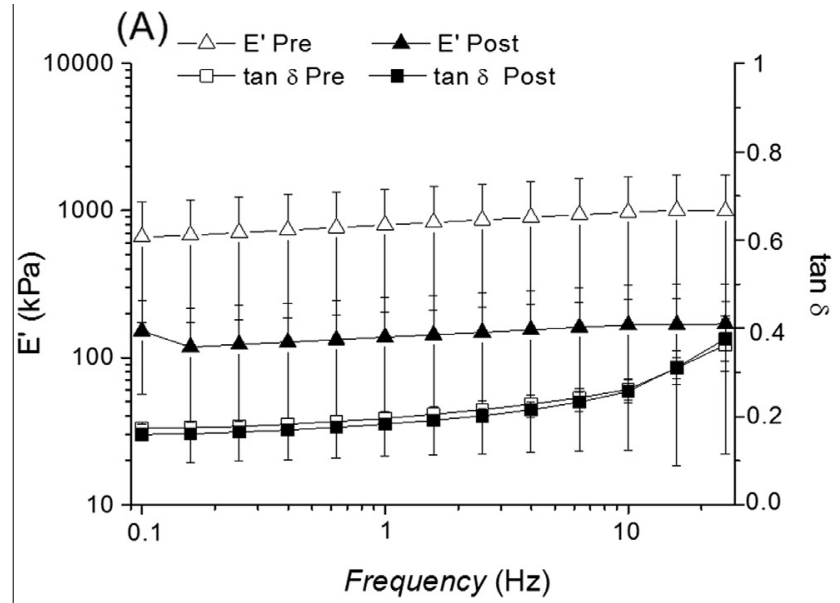

(C)

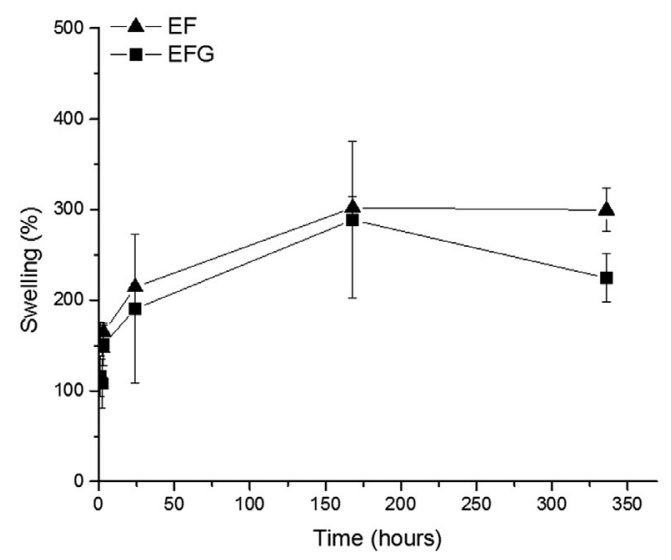

(B)

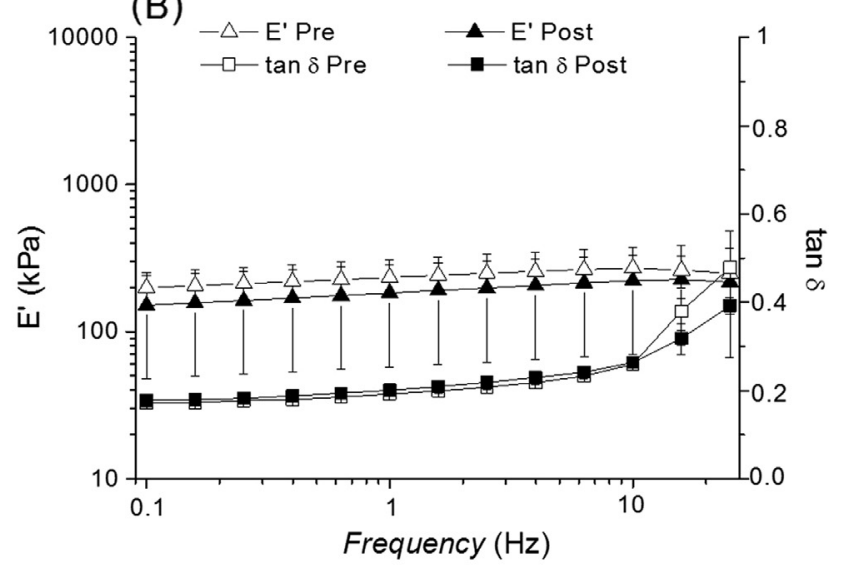

(D)

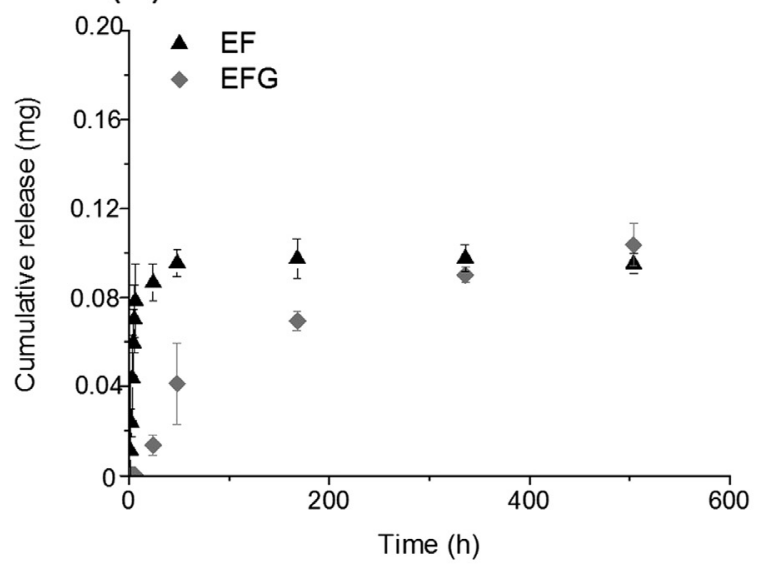

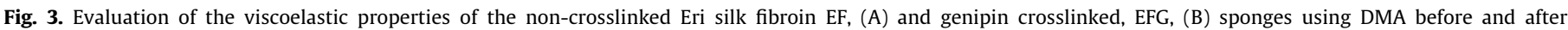

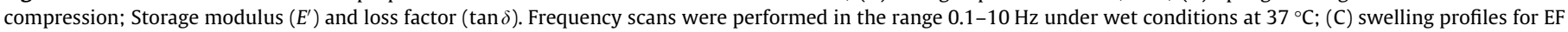

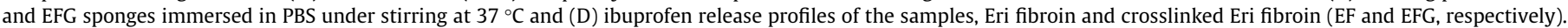

exhibit higher stiffness. Moreover, silk materials originating from $B$. mori have been reported to increase their $\beta$-sheet content after exposure to genipin [7]. In fact, an increase in the $\beta$-sheet content of EFG sponges (48.7\%) was observed, as compared to EF sponges (38.1\%). However, the decrease in stiffness of EFG sponges compared to EF sponges may be attributed to the flexibility conferred by the genipin crosslinks. The flexibility of such crosslinked structures is previously described by Mathew et al., using collagen structure crosslinked with genipin [47]. It is also worth noting that the exposure of the EFG sponges to a genipin-containing atmosphere led to interesting results regarding two different aspects: (i) the reproducibility of the sponges' mechanical properties (EF sponges showed a higher variability in $E^{\prime}$ values; while the mechanical properties of EFG sponges were possibly modulated due to the formation of an organized structure, richer in $\beta$-sheet content) and (ii) the ability of the sponges to recover from mechanical deformation, keeping their mechanical properties close to the original (pre-deformation) ones. After compression of $360 \mathrm{kPa}$, the EF and EFG sponges showed average recovery rates of $E^{\prime}$ value of $15 \%$ and $78 \%$, respectively. The compression of the sponges with $360 \mathrm{kPa}$ for $30 \mathrm{~s}$ aimed at assessing the ability of the materials to recover their initial height after application of compressive stress leading to high deformations. Moreover, we also aimed at tracing the changes in their mechanical properties after such procedure. This may provide especially important information in the case of handling of the sponges during surgeries. If they are capable of being deformed and occupy very low volume space, the sponges may be applied in the defect sites through minimally invasive strategies. After measuring the differences in the height of the sponges, before and during the $360 \mathrm{kPa}$ compression, the calculated deformations were in the order of $51.7 \pm 2.9 \%$ and $64.7 \pm 4.1 \%$ for $\mathrm{EF}$ and $\mathrm{EFG}$, respectively. While the $E^{\prime}$ values registered for EF sponges before and after compression were significantly different $(p<0.01)$, the values for EFG did not show statistical differences. Consequently, it can be said that EFG show a much more efficient maintenance in their mechanical properties after the application of the compression step, as compared to EF sponges. Such results indicates that, although the crosslinking reaction led to a decrease in the stiffness of the sponges; and despite the height recovery from compression of both types of sponges upon deformation (96 $\pm 6.2 \%$ and $93 \pm 6.1 \%$ for EF and EFG sponges, respectively), their recovery properties (regarding the maintenance of stiffness) were highly improved after exposure to ethanol vapor with dissolved genipin. This suggests that the covalent bonds and increase in the $\beta$-sheet flexible structure avoids the destruction of the EFG sponges' inner architecture upon the application of compressive deformations up to $50-60 \%$ of the original height of the sponges. The storage modulus $\left(E^{\prime}\right)$ of both types of sponges was weakly dependent on frequency over the entire range, as shown previously by woven silk membranes, prepared from the $B$. mori species [48]. The overall findings suggest that the developed sponges have recovery from compression character- 
istics. Specifically, EFG sponges show a high capability to recover their mechanical properties after the application of a compressive deformation.

The damping ability of the sponges can be evaluated by analyzing the $\tan \delta$ corresponding to $E^{\prime \prime} \mid E^{\prime}$ ratio. The $\tan \delta$ results indicate that the damping capability is not different for the two materials and does not depend on the frequency applied to the materials (Fig. 3B). Also, both EF and EFG presented loss factor values higher than 0.2 over the complete range of frequencies, which confirm their clear viscoelastic behavior, i.e. an ability to absorb mechanical energy [49].

\subsection{Swelling degree and drug release}

The swelling degree of the sponges immersed in PBS is shown in Fig. 3C. Both sponges presented an appreciable swelling degree. However, the results also revealed that the EFG has lower water uptake ability than EF. In EFG sponges, the introduced crosslinks create stable structures that restrict their swelling. We also observe that both sponges kept their 3D structure after 60 days, and the weight loss was $25.3 \pm 4.3 \%$ and $16.5 \pm 3.0 \%$ for EF and $\mathrm{EFG}$, respectively. The presence of the intermolecular crosslinked network in EFG structure increases their structure stability. According to the literature, the degradability of silk biomaterials depends on morphological features, processing methodologies and $\beta$-sheet content [50]. For this reason, these results suggest that the differences in $\beta$-sheet crystalline content within the matrices may have a significant role in their stability when incubated in PBS.
The characteristics of silk-based sponges could be interesting in the construction of a drug delivery system. For that purpose, ibuprofen, a nonsteroidal anti-inflammatory drug widely used for the treatment of different types of arthritis was used to analyze the release profile of such drug from the silk-based sponges [51]. We hypothesized that depending on the crosslinking extent of the material, the introduced crosslinks into EF chains will control the kinetics of release of the drug. The impregnation of ibuprofen in the sponges was made through immersion them overnight followed by: (i) frozen at $-80^{\circ} \mathrm{C}$ and freeze-drying and (ii) immersion in a genipin solution for $48 \mathrm{~h}$. The values for impregnation yield were $26.5 \pm 1.3 \%$ and $17.1 \pm 0.8 \%$ for EF and EFG, respectively. The differences between EF and EFG suggest that the interactions between ibuprofen and EF were stronger than the ones with EFG. Also, EFG after impregnation with ibuprofen and genipin crosslinking showed a crosslinking degree of $39.8 \pm 3.1 \%$, determined by ninhydrin assay $[29,30]$. Therefore, it is crucial to evaluate the drug release profile from the samples. The release profiles of ibuprofen from both types of sponges were evaluated in PBS for a period up to 3 weeks (Fig. 3D). The data indicate that the release of drug from EFG and EF have different kinetics. EFG sponges show slower release than EF sponges. This is in agreement with the hypothesis that the presence of crosslinked regions in the EFG samples can control the release kinetics of the drug. We hypothesize for EF sponges that the drug molecules are captured by EF chains during the structural transition. Similar behavior is observed by Dong et al. in their study on silk fibroin-coated liposomes for ocular drug delivery [52].
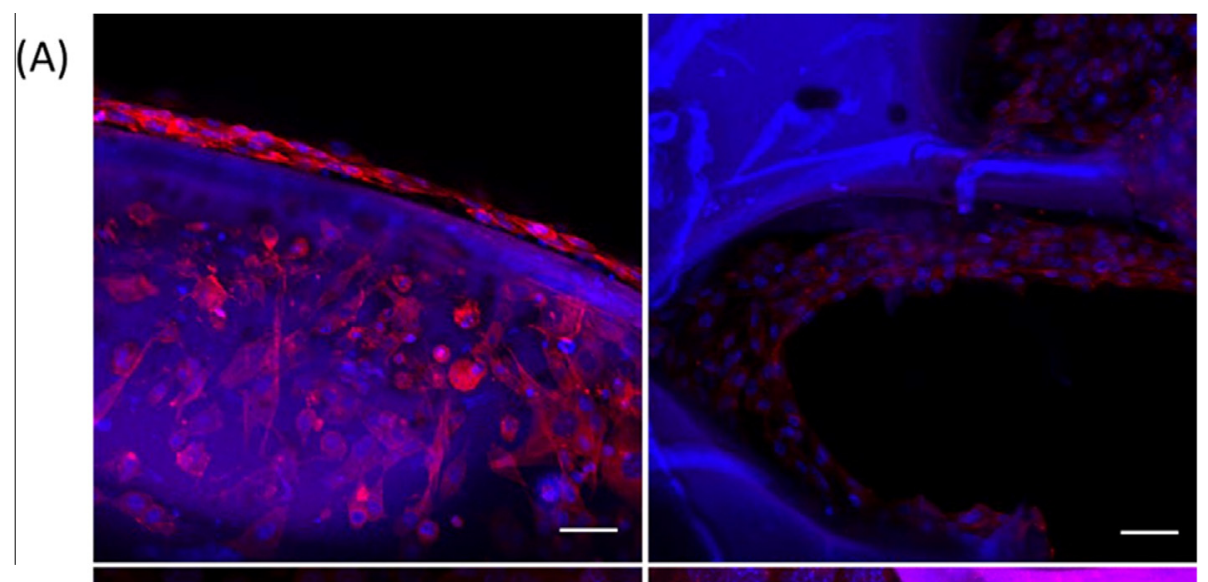

(B)
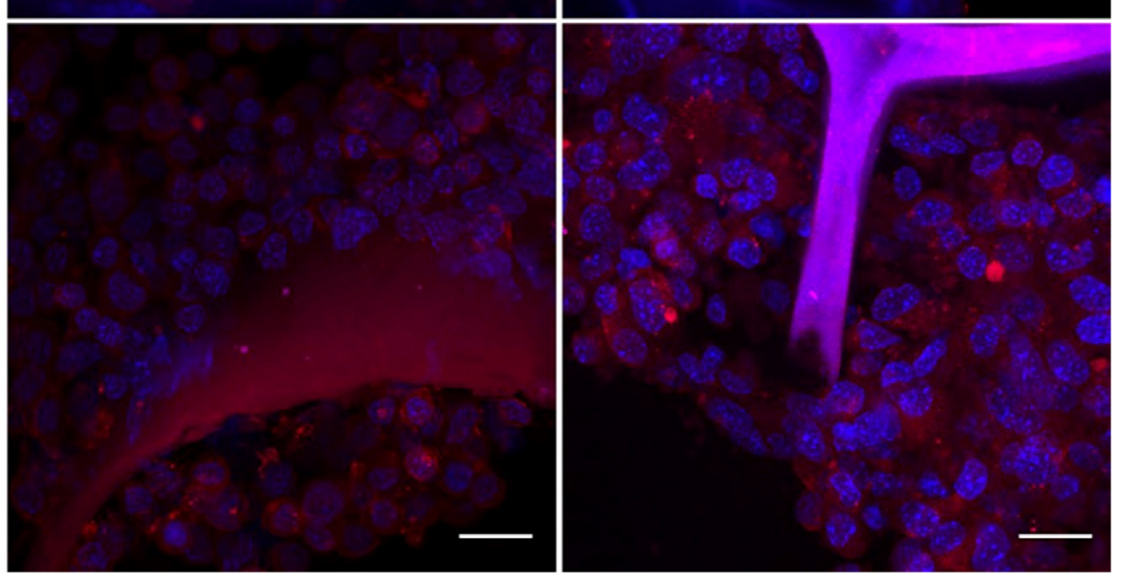

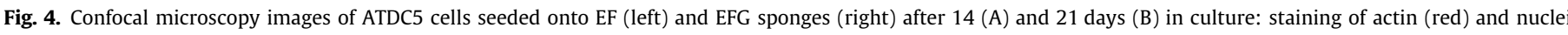

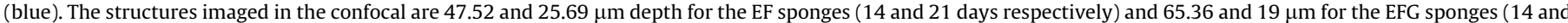

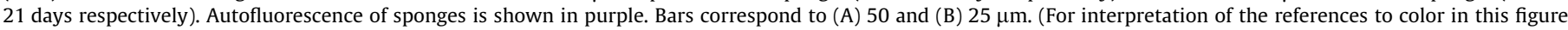
legend, the reader is referred to the web version of this article.) 
(A)

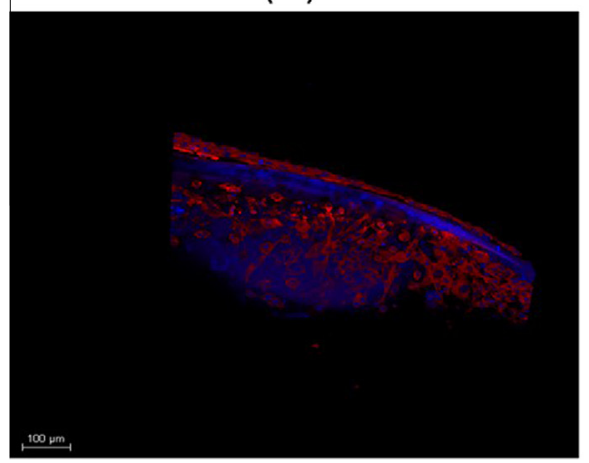

(B)

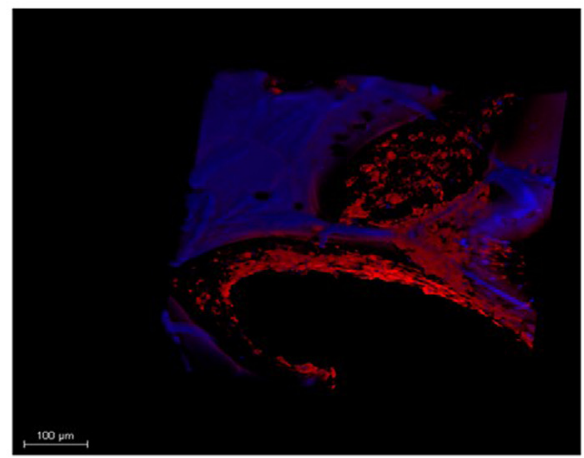

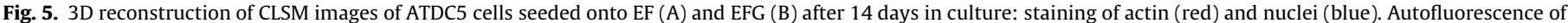
sponges is shown in purple. (For interpretation of the references to color in this figure legend, the reader is referred to the web version of this article.)

\subsection{Biological behavior}

ATDC5 attachment to the EF and EFG sponges was evaluated at different time points by confocal laser scanning microscopy (CLSM) (Fig. 4A). Images obtained by CLSM confirmed both cell attachment and spreading on the surface of the sponges (Fig. 4). Independently of the sponges' type, the cells attached to their surfaces and also inside the pores near the surface of the sponges. In both cases, after a cross-section analysis, few cells were observed inside the sponges, revealing an inhomogeneous cell distribution. We hypothesize that this fact could be attributed to the medium interconnectivity values found in the structures, despite the pore size compatible with cellular migration found in the structure of both type of sponges (see Table 1). In fact, scaffolds with higher pore size $(680 \mu \mathrm{m})$ and $100 \%$ interconnectivity showed inhomogeneous cell distribution and ECM deposition [53]. Regarding the zonal cell and ECM distribution in articular cartilage, this lack of homogeneous distribution of cells within the sponge could be a favorable aspect, given the fact that we could be recapitulating the natural environment, thus providing conditions for instructive cell and/or ECM distribution. In both EF and EFG sponges, ATDC5 cells spread well throughout the pores and show a flat morphology with an organized cytoskeleton after 14 days in culture (Fig. 4A). While some cells maintain the normal morphology of these cells in $2 \mathrm{D}$
(A)

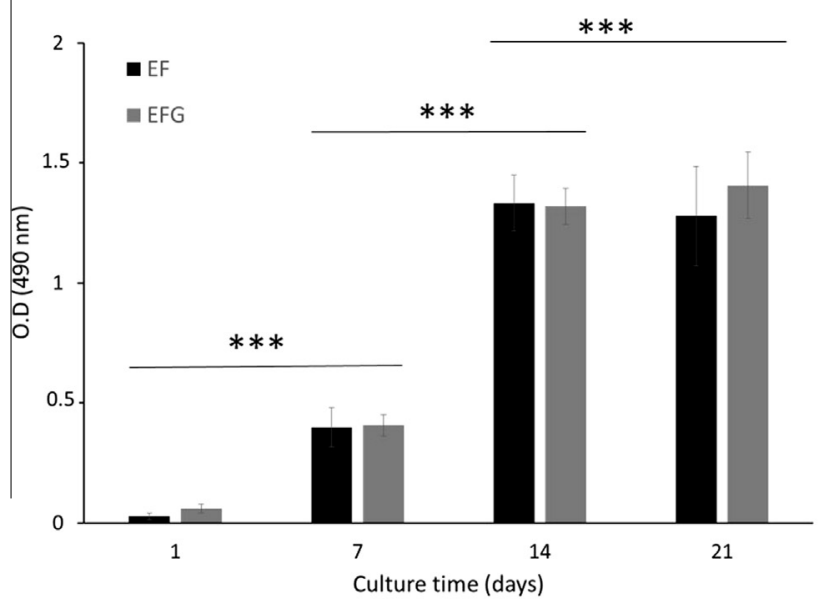

(B)

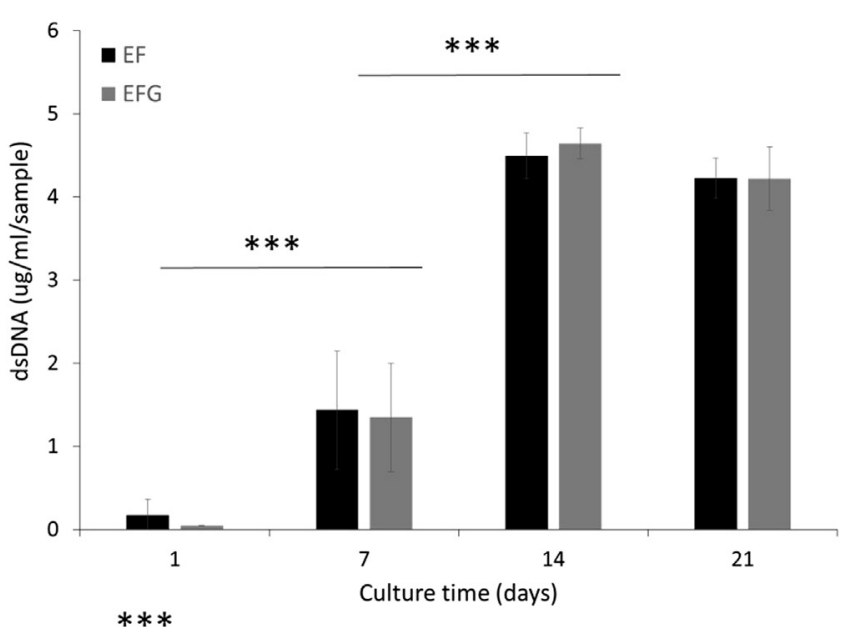

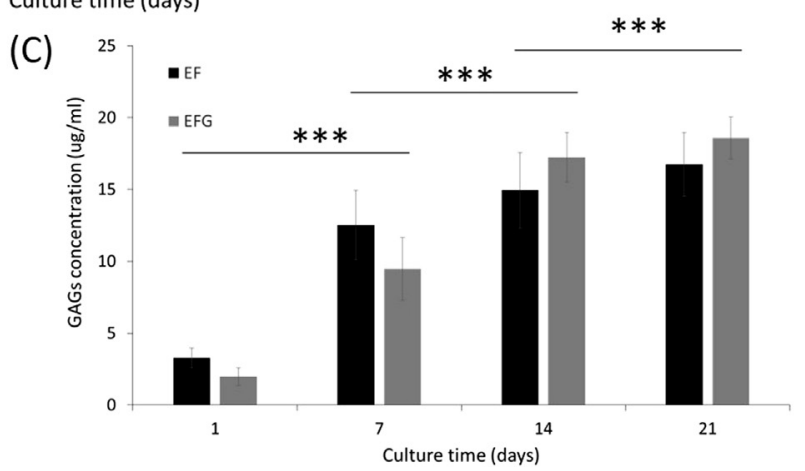

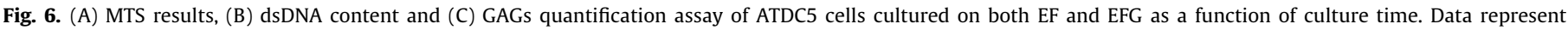
means \pm standard deviation $(p<0.001$, two-way ANOVA). 

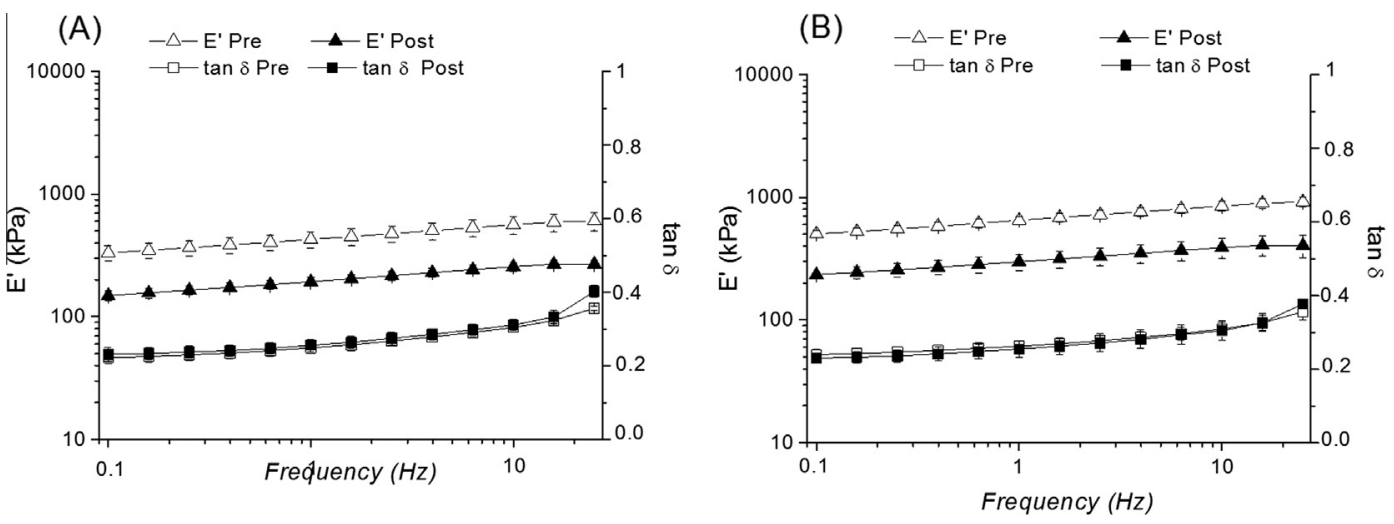

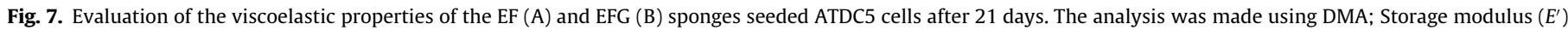
and loss factor $(\tan \delta)$. Frequency scans were performed in the range $0.1-10 \mathrm{~Hz}$ under wet conditions at $37^{\circ} \mathrm{C}$.

surfaces, other show round-shaped morphology resembling chondrocytes in a 3D environment (Fig. 4). The 3D reconstructions of images acquired near the surface of the sponge show that cells adhered to the interior of the pores (Fig. 5, in purple) and spread onto the surface of the sponge (Fig. 5).

The MTS results show an increase in ATDC5 cells metabolic activity with the time of culture, for both EF and EFG sponges (Fig. 6A). There is a significant increase $(p<0.001)$ in cell metabolic activity for EF and EFG until 14 days of culture. Results obtained from DNA biochemical analysis show a clear proliferation tendency of ATDC5 chondrocytes-like cells seeded onto EF and EFG sponges, with a tendency to increase with the culturing time $(p<0.001)$ (Fig. 6B). The results obtained by dsDNA quantification suggest that the increase in metabolic activity observed with increasing time of culture can be probably attributed to cell proliferation (Fig. 6A and B). In the two last time points we could not observe significant differences in terms of cell proliferation, which is in accordance with the microscopy results obtained. This shows no differences in cell density in both sponge formulations. The increase in metabolic activity and cell proliferation was accompanied by an increase in GAG content for both sponge compositions. Although statistical differences were not found after prolonged culture time (14 and 21 days), the results seem to indicate a tendency to increase the amount of extracellular matrix (ECM). This is produced in both formulations (Fig. 6C). In future approaches using mesenchymal stem cells, the analysis of common chondrocyte molecular markers may allow the confirmation of the chondrogenic lineage.

The mechanical properties of the constructs after 21 days of cell culture using ATDC5 cells were measured by DMA (Fig. 7). Both EF and EFG constructs showed values similar to native cartilage [54]. EFG sponges exhibited significantly higher $(p<0.05)$ storage modulus $\left(E^{\prime}\right)$ than $\mathrm{EF}$ before and after compression step. For a frequency of $1 \mathrm{~Hz}$, the values of storage modulus were $420 \mathrm{kPa}$ and $630 \mathrm{kPa}$ before compression, and $180 \mathrm{kPa}$ and $310 \mathrm{kPa}$ after compression, for EF and EFG, respectively. The extracellular matrix (ECM) formation evidenced by GAGs determination (Fig. 6C) may have a significant role in the observed differences in mechanical behavior between EF and EFG. Previous DMA studies performed with biomaterials, such as gellan gum for intervertebral disk [55] or kappa-carrageenan for cartilage repair [56] showed an increase in $E^{\prime}$ values after 3 weeks of cell culture with primary intervertebral disk cells or ATDC5 cells, respectively. The ECM produced by the chondrocyte cell line together with the weight loss of the sponges may explain these results. Although EF sponges show a higher $E^{\prime}$ value before the addition of cells, its weight loss in physiological-like solution was greater than the one for EFG sponges. It is important to highlight that after 21 days in culture, both sponges kept the shape recovery characteristics upon hydration with PBS ( $99.6 \pm 1.7 \%$ for EF and $96.6 \pm 1.7 \%$ for EFG). Interestingly, both the materials show the same average recovery rate regarding $E^{\prime}$ values: $45 \%$. These results led to the hypothesis that after 21 days of cell culture on the sponges, the recovery rate of the mechanical properties after compression may mainly be determined by the production of ECM in the sponges since they show very distinct behavior before cell culture.

\section{Conclusions}

The present approach provides a promising avenue to produce silk fibroin non-crosslinked and genipin crosslinked silk sponges from non-mulberry semi domesticated Eri silk cocoons of S. ricini. After applying $50-60 \%$ of deformation on the biomaterials, the recovery analysis shows that before the cell culture the sponges have high size recovery ability: $96 \%$ and $93 \%$ for EF and EFG sponges, respectively. These features indicate some advantages such as biomaterial flexibility to adapt and recover their shapes according to the defect sites where it will be implanted. This may be applicable even after exposure to high deformations, which may occur during surgical procedure. The developed matrices may be used as controlled drug release system as demonstrated through the incorporation and release of ibuprofen. In vitro biological performance of the sponges with ATDC5 chondrocyte-like cells reveals a positive effect of the structures over cell adhesion, proliferation and ECM production. Interestingly, the sponges kept their recovery properties after 21 days of cell culture, while the mechanical properties seem to be dictated by the production of ECM. The findings suggest that the developed Eri cocoon silk fibroin sponges may be a good candidate for cartilage-related biomedical applications.

\section{Notes}

The authors declare no competing financial interest.

\section{Acknowledgments}

The authors SSS, DSC, MBO, NMO acknowledge financial support from Portuguese Foundation for Science and Technology FCT (Grants SFRH/BPD/45307/2008, SFRH/BPD/85790/2012, SFRH/BD/71396/2010 and SFRH/BD/73172/2010, respectively), "Fundo Social Europeu" - FSE, and "Programa Diferencial de Potencial Humano POPH". This work is also financially supported by the 
European Union Seventh Framework Programme (FP7/2007-2013) under grant agreement $\mathrm{n}^{\circ}$ REGPOT-CT2012-316331-POLARIS and from Fundação para a Ciência e Tecnologia (FCT) through the project ENIGMA - PTDC/EQU-EPR/121491/2010. The laboratory work of SCK is supported by Department of Biotechnology and Indian Council of Medical Research, Govt of India. SCK and RLR acknowledge their short visits either Institutes. SCK is also grateful to 3B́s Research Group- Biomaterials, Biodegradables and Biomimetics, University of Minho, Portugal for providing facilities during his short visit.

\section{References}

[1] M. Silva, A. Martins, A. Teixeira, R.L. Reis, N. Neves, Impact of biological agents and tissue engineering approaches on the treatment of rheumatic diseases, Tissue Eng. Part B: Rev. 16 (2010) 331-339.

[2] J.F. Mano, R.L. Reis, Osteochondral defects: present situation and tissue engineering approaches, J. Tissue Eng. Regen. Med. 1 (2007) 261-273.

[3] S.S. Silva, J.M. Oliveira, H. Sá-Lima, R.A. Sousa, J.F. Mano, R.L. Reis, Polymers of biological origin, in: P. Ducheyne (Ed.), Comprehensive Biomaterials, Elsevier Oxford, 2011, pp. 187-205.

[4] J.F. Mano, G.A. Silva, H.S. Azevedo, P.B. Malafaya, R.A. Sousa, S.S. Silva, et al. Natural origin biodegradable systems in tissue engineering and regenerative medicine: present status and some moving trends, J. R. Soc. Interface 4 (2007) 999-1030.

[5] D.N. Rockwood, R.C. Preda, T. Yucel, X. Wang, M.L. Lovett, D.L. Kaplan, Materials fabrication from Bombyx mori silk fibroin, Nat. Protocols 6 (2011) 1612-1631.

[6] W. Sun, T. Incitti, C. Migliaresi, A. Quattrone, S. Casarosa, A. Motta, Genipincrosslinked gelatin-silk fibroin hydrogels for modulating the behaviour of pluripotent cells, J. Tissue Eng. Regen. Med. (2014), http://dx.doi.org/10.1002/ term.1868.

[7] S. Silva, A. Motta, M.T. Rodrigues, A. Pinheiro, M. Gomes, J. Mano, et al., Novel genipin-cross-linked chitosan/silk fibroin sponges for cartilage engineering strategies, Biomacromolecules 9 (2008) 2764-2774.

[8] N. Bhardwaj, S.C. Kundu, Chondrogenic differentiation of rat MSCs on porous scaffolds of silk fibroin/chitosan blends, Biomaterials 33 (2012) 2848-2857.

[9] S.C. Kundu, B. Kundu, S. Talukdar, S. Bano, S. Nayak, J. Kundu, et al., Nonmulberry silk biopolymers, Biopolymers 97 (2012) 455-467.

[10] S. Kar, S. Talukdar, S. Pal, S. Nayak, P. Paranjape, S.C. Kundu, Silk gland fibroin from Indian Muga silkworm Antheraea assama as potential biomaterial, TERM 10 (2013) 200-210.

[11] S. Saha, B. Kundu, J. Kirkham, D. Wood, S.C. Kundu, X.B.B. Yang, Osteochondral tissue engineering in vivo: a comparative study using layered silk fibroin scaffolds from mulberry and nonmulberry silkworms, PLoS One 8 (2013), http://dx.doi.org/10.1371/journal.pone.0080004.

[12] S. Talukdar, S.C. Kundu, Engineered 3D silk-based metastasis models: interactions between human breast adenocarcinoma, mesenchymal stem cells and osteoblast-like cells, Adv. Funct. Mater. 23 (2013) 5249-5260.

[13] B. Kundu, S.C. Kundu, Bio-inspired fabrication of fibroin cryogels from the muga silkworm Antheraea assamensis for liver tissue engineering, Biomed. Mater. 8 (2013)

[14] S. Pal, J. Kundu, S. Talukdar, T. Thomas, S.C. Kundu, An emerging functional natural silk biomaterial from the only domesticated non-mulberry silkworm Samia ricini, Macromol. Biosci. 13 (2013) 1020-1035.

[15] Y. Nakazawa, M. Bamba, S. Nishio, T. Asakura, Tightly winding structure of sequential model peptide for repeated helical region in Samia cynthia ricini silk fibroin studied with solid-state NMR, Protein Sci. 12 (2003) 666-671.

[16] C. Vepari, D.L. Kaplan, Silk as a biomaterial, Prog. Polym. Sci. 32 (2007) 991 1007.

[17] S. Dutta, B. Talukdar, R. Bharali, R. Rajkhowa, D. Devi, Fabrication and characterization of biomaterial film from gland silk of muga and eri silkworms, Biopolymers 99 (2013) 326-333.

[18] D.M. Phillips, L.F. Drummy, D.G. Conrady, D.M. Fox, R.R. Naik, M.O. Stone, et al., Dissolution and regeneration of Bombyx mori silk fibroin using ionic liquids, J. Am. Chem. Soc. 126 (2004) 14350-14351.

[19] S.S. Silva, E.G. Popa, M.E. Gomes, M.B. Oliveira, S. Nayak, B. Subia, et al., Silk hydrogels from non-mulberry and mulberry silkworm cocoons processed with ionic liquids, Acta Biomater. 9 (2013) 8972-8982.

[20] S.S. Silva, T.C. Santos, M.T. Cerqueira, A.P. Marques, L.L. Reys, T.H. Silva, et al. The use of ionic liquids in the processing of chitosan/silk hydrogels for biomedical applications, Green Chem. 14 (2012) 1463-1470.

[21] N. Goujon, X. Wang, R. Rajkowa, N. Byrne, Regenerated silk fibroin using protic ionic liquids solvents: towards an all-ionic-liquid process for producing silk with tunable properties, Chem. Commun. 48 (2012) 1278-1280.

[22] N. Goujon, R. Rajkhowa, X. Wang, N. Byrne, Effect of solvent on ionic liquid dissolved regenerated Antheraea assamensis silk fibroin, J. Appl. Polym. Sci. 128 (2013) 4411-4416.

[23] F.-L. Mi, H.-W. Sung, S.-S. Shyu, Synthesis and characterization of a novel chitosan-based network prepared using naturally occurring crosslinker, Polym. Sci. A Polym. Chem 38 (2000) 2804-2814.

[24] H.-W. Sung, Y. Chang, I.L. Liang, W.-H. Chang, Y.-C. Chen, Fixation of biological tissues with a naturally occurring crosslinking agent: Fixation rate and effects of $\mathrm{pH}$, temperature, and initial fixative concentration, J. Biomed. Mater. Res. 52 (2000) 77-87.

[25] W.H. Elliott, W. Bonani, D. Maniglio, A. Motta, W. Tan, C. Migliaresi, Silk hydrogels of tunable structure and viscoelastic properties using different chronological orders of genipin and physical cross-linking, ACS Appl. Mater. Interfaces 7 (2015) 12099-12108.

[26] X. Deng, L. Mammen, H.-J. Butt, D. Vollmer, Candle soot as a template for a transparent robust superamphiphobic coating, Science 335 (2012) 67-70.

[27] M.I. Rial-Hermida, N.M. Oliveira, A. Concheiro, C. Alvarez-Lorenzo, J.F. Mano, Bioinspired superamphiphobic surfaces as a tool for polymer- and solventindependent preparation of drug-loaded spherical particles, Acta Biomater. 10 (2014) 4314-4322.

[28] A.M.S. Costa, M. Alatorre-Meda Manuel Oliveira Nuno, J.F. Mano, Biocompatible polymeric microparticles produced by a simple biomimetic approach, Langmuir 30 (2014) 4535-4539.

[29] M. Friedman, Applications of the ninhydrin reaction for analysis of amino acids, peptides, and proteins to agricultural and biomedical sciences, J. Agric. Food Chem. 52 (2004) 385-406.

[30] Y. Yuan, B.M. Chesnut, G. Utturkar, W.O. Haggard, Y. Yang, J.L. Ong, et al., The effect of cross-linking of chitosan microspheres with genipin on protein release, Carbohydr. Polym. 68 (2007) 561-567.

[31] X. Hu, D. Kaplan, P. Cebe, Determining beta-sheet crystallinity in fibrous proteins by thermal analysis and infrared spectroscopy, Macromolecules 39 (2006) 6161-6170.

[32] W. Kafienah, T.J. Sims, Biochemical methods for the analysis of tissueengineered cartilage, in: A.P. Hollander, P.V. Hatton (Eds.), Biopolymer Methods in Tissue Engineering, New York Human Press Incorporation, 2004, pp. 217-229.

[33] X. Hu, K. Shmelev, L. Sun, E.-S. Gil, S.-H. Park, P. Cebe, et al., Regulation of silk material structure by temperature-controlled water vapor annealing, Biomacromolecules 12 (2011) 1686-1696.

[34] J. Anthony, J. Brennecke, J. Holbrey, E. Maginn, R. Mantz, R. Rogers, et al. Physicochemical properties of ionic liquids, in: P.W.T. Wasserscheid (Ed.), Ionic Liquids in Synthesis, Wiley-VCH Verlag, Weinheim, 2003, pp. 41-126.

[35] S.S. Silva, A.R.C. Duarte, A.P. Carvalho, J.F. Mano, R.L. Reis, Green processing of porous chitin structures for biomedical applications combining ionic liquids and supercritical fluid technology, Acta Biomater. 7 (2011) 1166-1172.

[36] S.S. Silva, A.R.C. Duarte, J.F. Mano, R.L. Reis, Design and functionalization of chitin-based microsphere scaffolds, Green Chem. 15 (2013) 3252-3258.

[37] I.C. Um, H. Kweon, Y.H. Park, S. Hudson, Structural characteristics and properties of the regenerated silk fibroin prepared from formic acid, Int. J. Biol. Macromol. 29 (2001) 91-97.

[38] R. Rajkhowa, L. Wang, J.R. Kanwar, X. Wang, Molecular weight and secondary structure change in eri silk during alkali degumming and powdering, J. Appl. Polym. Sci. 119 (2011) 1339-1347.

[39] N.V. Bhat, G.S. Nadiger, Crystallinity in silk fibers: partial acid hydrolysis and related studies, J. Appl. Polym. Sci. 25 (1980) 921-932.

[40] A. Muthumanickkam, S. Subramanian, M. Goweri, W. Sofi Beaula, V. Ganesh, Comparative study on eri silk and mulberry silk fibroin scaffolds for biomedical applications, Iran. Polym. J. 22 (2013) 143-154.

[41] M. Andiyappan, S. Sundaramoorthy, P. Vidyasekar, N.T. Srinivasan, R.S. Verma, Characterization of electrospun fibrous scaffold produced from Indian eri silk fibroin, Int. J. Mater. Res. 104 (2013) 498-506.

[42] R.A.A. Muzzarelli, Genipin-crosslinked chitosan hydrogels as biomedical and pharmaceutical aids, Carbohydr. Polym. 77 (2009) 1-9.

[43] Structural aspects of silk, in: K.M. Babu (Ed.), Silk: Processing, Properties and Applications, Woodhead Publishing Series in Textiles, 2013, pp. 56-81.

[44] M. Li, Z. Wu, C. Zhang, S. Lu, H. Yan, D. Huang, et al., Study on porous silk fibroin materials. II. Preparation and characteristics of spongy porous silk fibroin materials, J. Appl. Polym. Sci. 79 (2001) 2192-2199.

[45] H.M. Aydin, A.J. El-Haj, E. Pişkin, Y. Yang, Improving pore interconnectivity in polymeric scaffolds for tissue engineering, J. Tissue Eng. Regen. Med. 3 (2009) 470-476.

[46] S. Sosnowski, P. Woźniak, M. Lewandowska-Szumieł, Polyester scaffolds with bimodal pore size distribution for tissue engineering, Macromol. Biosci. 6 (2006) 425-434

[47] A.P. Mathew, K. Oksman, D. Pierron, M.-F. Harmand, Biocompatible fibrous networks of cellulose nanofibres and collagen crosslinked using genipin: potential as artificial ligament/tendons, Macromol. Biosci. 13 (2013) 289-298.

[48] L.R. Almeida, A.R. Martins, E.M. Fernandes, M.B. Oliveira, V.M. Correlo, I. Pashkuleva, et al., New biotextiles for tissue engineering: development, characterization and in vitro cellular viability, Acta Biomater. 9 (2013) 8167-8181.

[49] M. Brodt, L.S. Cook, R.S. Lakes, Apparatus for measuring viscoelastic properties over ten decades: refinements, Rev. Sci. Instrum. 66 (1995) 5292-5297.

[50] R. You, Y. Xu, G. Liu, Y. Liu, X. Li, M. Li, Regulating the degradation rate of silk fibroin films through changing the genipin crosslinking degree, Polym. Degrad. Stab. 109 (2014) 226-232.

[51] A. Carballido, R. Vanrell, M. Martinez, P. Pastoriza, Biodegradable ibuprofenloaded PLGA microspheres for intraarticular administration Effect of Labrafil addition on release in vitro, Int. J. Pharm. 279 (2004) 33-41.

[52] Y. Dong, P. Dong, D. Huang, L. Mei, Y. Xia, Z. Wang, et al., Fabrication and characterization of silk fibroin-coated liposomes for ocular drug delivery, Eur. J. Pharm. Biopharm. 91 (2015) 82-90. 
[53] T.B.F. Woodfield, C. Van Blitterswijk, J. De Wijn, T. Sims, A. Hollander, J. Riesle, Polymer scaffolds fabricated with pore-size gradients as a model for studying the zonal organization within tissue-engineered cartilage constructs, Tissue Eng. 11 (2005) 1297-1311.

[54] G. Fulcher, D. Hukins, D. Shepherd, Viscoelastic properties of bovine articular cartilage attached to subchondral bone at high frequencies, BMC Musculoskelet. Disord. 10 (2009) 61, http://dx.doi.org/10.1371/journal. pone.0080004.
[55] J. Silva-Correia, A. Gloria, M.B. Oliveira, J.F. Mano, J.M. Oliveira, L. Ambrosio, et al., Rheological and mechanical properties of acellular and cell-laden methacrylated gellan gum hydrogels, J. Bio. Mater. Res. A 101 (2013) 34383446.

[56] E.G. Popa, M.T. Rodrigues, D.F. Coutinho, M.B. Oliveira, J.F. Mano, R.L. Reis, et al., Cryopreservation of cell laden natural origin hydrogels for cartilage regeneration strategies, Soft Matter 9 (2013) 875-885. 